\title{
Mini invasive approaches in the treatment of small renal masses: TC-guided renal cryoablation in elderly
}

\author{
Oscar Selvaggio $^{1}$, Giovanni Silecchia ${ }^{1}$, Matteo Gravina ${ }^{2}$, Ugo Giovanni Falagario ${ }^{1}$, Giovanni Stallone ${ }^{3}$, \\ Luca Macarini $^{2}$, Giuseppe Carrieri ${ }^{1}$, Luigi Cormio ${ }^{1,4}$ \\ ${ }^{1}$ University of Foggia, Department of Urology and Organ transplantation, Foggia, Italy; \\ ${ }^{2}$ University of Foggia, Department of Radiology, Foggia, Italy; \\ ${ }^{3}$ University of Foggia, Department of nephrology, Foggia, Italy; \\ ${ }^{4}$ Department of Urology, Bonomo Teaching Hospital, Andria (BAT), Italy.
}

\begin{abstract}
Summary Background: Today, the goal of surgery is to achieve oncological efficacy with the lowest complication rate. Computed Tomography (CT)-guided cryoablation is proposed as a safe and effective technique. We report, our series of small renal masses treated with cryoablation in elderly ( $>70$ years).

Methods: From May 2014 to April 2019, 32 patients with median (IQR) age of 75.5 years (range 71-80) with small renal masses (<T1a) diagnosis, clinical anesthesia contraindications to nephron-sparing surgery or patient's will previous informed consent have been selected at our Urology Department. All patients underwent CT-guided cryoablation, preceded by needle biopsy. The cryoablation consisted in a procedure with an argon/helium gas-based system under local anesthesia. The follow-up included CT abdomen at 3, 6 and 12 months. The definition of incomplete treatment was the persistence of the lesion contrast enhancement (CE) at the end of the scan; the definition of relapse was the appearance of the CE to the 6-month control CT.

Results: The median follow-up was 30 months (IQR 1-59). The median size of the tumor was $3.85 \mathrm{~cm}$ (IQR 1.6-4.5). All patients underwent lesion biopsy resulting in diagnosis of Renal Cell Carcinoma (RCC) in 29 patients (90.7\%) and oncocytoma in 3 patients (9.3\%). A median of 2 cryoprobes (IQR 13) was used and 2/3 cycles of freeze-thaw of the duration of 10 minutes or 5 minutes were performed. Complications were: 3 asymptomatic transitional perirenal effusion, 2 lumbar pain well-controlled by analgesic drug. Hospital stay was 2 days (range 1-3). No case showed incomplete treatment and local relapse or metastates at the CT abdomen-pelvis with contrast medium at 12 months.

Conclusions: This study shows the efficacy and safety of percutaneous cryoablation of small renal masses in elderly population. The procedure is easy to perform, with low complication rates and well tolerated by the elderly patients.
\end{abstract}

KEY WORDS: Percutaneous renal cryoablation; Kidney cancer; Small renal masses; Elderly; Mini invasive.

Submitted 10 May 2020; Accepted 10 June 2020

\section{INTRODUCTION}

Extensive application of modern imaging techniques has led to more frequent incidental diagnosis of small renal masses (SMRs) (1-3) with an increasing incidence by an average of $3 \%$ to $4 \%$ every year $(3,4)$. Kidney cancer represents today $3 \%$ of all tumors diagnosed in male and female over 70 years old (5).

A growing body of evidence support the importance of treatments preserving kidney function $(6,7)$.

Additionally, since $25 \%$ of SMRs are benign cortical tumors (eg. Oncocytoma and angiomyolipoma) and another 25\% are indolent with limited metastatic potential (eg. Chromophobe, type 1 papillary renal cancer), several options for management of clinically localized renal masses have been reported, including active surveillance, thermal ablation and surgery that include radical and partial nephrectomy (8). Active surveillance is an option for patients presenting small renal masses $(<4$ $\mathrm{m}$, clinical stage $\mathrm{Tla}$ ) with a low likelihood of aggressive malignancy, procedure-limiting comorbidity, and/or limited life-expectancy (9). Nephron-sparing surgery (NSS) is considered the gold standard treatment for renal cell carcinoma (RCC) preferred for lesions less than $7 \mathrm{~cm}$ in diameter (clinical stage T1) (9).

In order to of decrease pain, morbidity, kidney function damages, hospital stay, and operative time, various minimally invasive modalities for NSS have been evaluated ( 6 , 7, 10-12). Thermal ablation, which may include cryoablation or radiofrequency ablation, may be used for small clinically localized masses (clinical stage Tla) and can be performed laparoscopically or percutaneously (9).

EAU guidelines suggest active surveillance, radiofrequency ablation and cryoablation for the treatment of elderly and/or comorbid patients with small renal masses, however the quality of the available data does not allow definitive conclusions regarding morbidity and oncological outcomes of cryoablation and radiofrequency ablation and the Strength rating of this recommendation remain week (9). The aim of this study was to present our initial experience in terms of efficacy and safety of CT-guided percutaneous cryoablation for small renal masses.

\section{MATERIALS AND METHOdS}

Study population

After Istitutional review board approval we retrospectively reviewed our prospectively kidney cancer database to evaluate outcomes of patients treated with CT- 
guided percutaneous cryoablation. The following inclusion criteria were used: tumor diameter $<4 \mathrm{~cm}$ (Tla); comorbidity or clinical anesthesia contraindications to nephron-sparing surgery; residual renal masses in patients already undergoing surgery; kidney solitary condition; informed consent. Exclusion criteria were lack of percutaneous access window, disease extending into renal vein or invading adjacent organs and tumor larger than $4 \mathrm{~cm}$ in a candidate for surgical treatment.

All patients were seen in the clinic before ablation, history was recorded, physical examination was performed, relevant baseline laboratory values were measured, and dual-phase renal CT imaging was performed. All patients were counseled regarding all the treatment options available, after that a written informed consent was obtained.

\section{CT-guided percutaneous cryoablation}

All procedures were performed by the same team of urologists and interventional radiologists on a specific TC General Electric Medical System Brightspeed. All patients were premedicated intravenously with 2 g cefazolin and ciprofloxacin $400 \mathrm{mg}$ versus cotrimoxazole 2 $\mathrm{fl}$ in the case of allergy. Patients were positioned prone or side, to facilitate probe insertion through a better percutaneous access window; for example, caused by descent of adjacent organs most often represented by bowel, on the CT bed and the ipsilateral flank was prepared and draped in a sterile fashion. A pre-procedural non-enhanced CT image was obtained to document tumor size and decide on access, number of probes and need for thermal protection for adjacent organs (hydrodissection). CT fluoroscopy was used to determine the optimum access site, with the help of a CT-mounted laser beam. The skin and underlying tissues were anesthetized with $10-15 \mathrm{ml}$ of lidocaine $200 \mathrm{mg}$, then a tiny incision (2-3 $\mathrm{mm}$ ) was made. Before percutaneous cryoablation, all the patients underwent CT-guided needle biopsy of the lesion according to the current recommendation and at least 2 good quality biopsy cores using an 18G needle were taken (9). The cryoprobes were then inserted according to preoperative plans in order to create an "iceball" that would cover the lesion and provide at least $5 \mathrm{~mm}$ of ablation beyond the tumor margins. The cryoprobe advancement toward the mass and the extent of the iceball was monitorated intermittently with CT fluoroscopy. The tip of probe was positioned through the mass until to distal inner border of the lesion. The operations were performed using Argon/helium gas-based system (Endocare, HealthTonics Inc., Austin TX, USA). Such system uses a compressed argon for freezing and compressed helium for active thawing. With two exceptions, in which $1.7 \mathrm{~mm}$ diameter cryoprobes were used, all procedures were performed with $2.4 \mathrm{~mm}$ diameter cryoprobes. The number of cryoprobes used varied as a function of the lesion's size: 8 patients treated with 1 probe, 18 pts with 2 probes, 6 pts with 3 probes. In seven cases, we have had a non-target organ (usually colon) that was closer than $1 \mathrm{~cm}$ to the tumor margin. In these cases, a Chiba's needle was inserted in the "fascia" of Gerota and sterile water, mixed to 5-7 cc of contrast medium, was infused to keep the non-target organ away from the iceball.
Technical success was defined as completion of three ablation cycle: a 10-minutes freeze, 10-minutes active thaw and 10-minutes repeat freeze with the iceball covering the entire lesion and extending at least $5 \mathrm{~mm}$ beyond its border.

At the end of the procedure, a CT image with contrast medium was performed to evaluate the complete cover of the lesion and possible local bleeding. The definition of incomplete treatment is the persistence of the CE at the end of the same. The patient was transferred to the recovery area and kept under observation. A blood count was performed 6 hour after the procedure and on postop day 1 before discharge. Patients were discharged home unless overnight observation was deemed necessary as a result of complications or severity of symptoms.

Complications were recorded using the Clavien-Dindo Classification and Grade I and II complications were

\section{Follow-up visits}

Follow-up visits were scheduled at 3-, 6-, 12-, 18-, 24and 36- months after the procedure. A physical examination was performed along with measurement of relevant laboratory values. Additionally, a dual-phase renal CT study was obtained at each follow-up visit. In order to minimize X-Ray exposure follow-up images were acquired using a Low dose protocol (low kilovolt, low milliampere). Additionally, only sequences of the treatment region were taken during the procedure and at follow-up. All studies were read by the same interventional radiologist and compared to the baseline study. Nonenhancement of the cryolesion was considered the primary radiographic hallmark of successful cryoablation. Response to treatment was based on tumor enhancement and size on sequential images. Complete lack of enhancement of a previously enhancing mass was considered complete response. Response based on tumor size was less reliable initially, as many masses did not change in size on the first 3 months imaging study despite a gradual decrease thereafter. The existence of enhancement areas in the residual tumor mass after 6 months was considered an indication of local failure.

\section{Statistical analysis}

Outcomes of this study were cancer recurrence, metastasis and complications after percutaneous cryoablation. Descriptive statistics was performed for the overall population. Continuous variables were reported as median and interquartile whereas categorical variables were reported as rates.

Statistical analyses were performed using Stata-SE 14 (StataCorp LP, College Station, TX, USA).

\section{Results}

Preoperative characteristics of the study population are shown in Table 1.

Final population included 20 men and 12 women with a median (IQR) age of 75.5 years (range $71-80$ years) treated at our institution between May 2014 to April 2019. The median (IQR) size of the tumors was $3.85 \mathrm{~cm}$ (range 1.6-4.5). Of the 32 treated patients, 30/32 (93.7\%) had an incidentaloma and 2/32 (6.3\%) were post-radiofre- 
Table 1.

Patient demographics and tumor characteristics of 32 patients.

\begin{tabular}{|c|c|}
\hline Variable & Cryoablation $n=32$ \\
\hline Age, years & $75.5(71-80)$ \\
\hline Tumor diameter, cm & $3.85(1.6-4.5)$ \\
\hline \multicolumn{2}{|l|}{ Gender, $n(\%)$} \\
\hline Male & $20(62.5 \%)$ \\
\hline Female & $12(37.5 \%)$ \\
\hline \multicolumn{2}{|l|}{ Side } \\
\hline Right & $14(43.7 \%)$ \\
\hline Left & $18(56.3 \%)$ \\
\hline \multicolumn{2}{|l|}{ ASA score n (\%) } \\
\hline 1 & $6(18.8 \%)$ \\
\hline 2 & $21(65.6 \%)$ \\
\hline 3 & $5(15.6 \%)$ \\
\hline \multicolumn{2}{|l|}{ Tumor location, n (\%) } \\
\hline Upper & $8(25.0 \%)$ \\
\hline Middle & $11(34.4 \%)$ \\
\hline Lower & $13(40.6 \%)$ \\
\hline \multicolumn{2}{|l|}{ Diagnosis, n (\%) } \\
\hline Incidentaloma & $30(93.7 \%)$ \\
\hline Radiofrequency failure & $2(6.3 \%)$ \\
\hline
\end{tabular}

Table 2.

Perioperative and postoperativecharacteristics.

\begin{tabular}{|l|c|}
\hline Variable & Cryoablation $\mathbf{n}=\mathbf{3 2}$ \\
\hline Hospital stay (d) & $2(1-3)$ \\
\hline Hystological outcomes to biopsy, $\mathbf{n}(\%)$ & $20(62.5 \%)$ \\
Clear cells RCC & $3(9.3 \%)$ \\
Chromophobe RCC & $6(18.7 \%)$ \\
Type I Papillary RCC & $3(9.3 \%)$ \\
Oncocytoma & $30(8-59)$ \\
\hline Follow-up, months & $5(15.6 \%)$ \\
Complications, $n(\%)$ & $0(0.0 \%)$ \\
Minor & $0(0.0 \%)$ \\
Major & $0(0.0 \%)$ \\
\hline Recurrence of tumor & \\
\hline Metastasis & \\
\hline
\end{tabular}

quency ablation relapses. Biopsy histological findings were the following: clear cell Renal Cell Carcinoma (RCC) 20/32 (62.5\%); chromophobe RCC 3/32 (9.3\%); Type 1 papillary $6 / 32$ (18.7\%); oncocytoma $3 / 32$ (9.3\%) (Table 2).

An average 2 cryoprobes (IQR: 1-3) have been used, depending on the size of the tumor, and were performed 2 cycles of freeze-thaw of the duration of 10 minutes each in 25 patients, and 3 cycles of 5 minutes each in 7 patients. 1.7 and $2.4 \mathrm{~mm}$ cryoprobes were used to minimize injury to the colon, we used thermal protective maneuvers in seven cases (21.8\%). There were no cases of non-target organ damage. Complications were: 3 effusion not clinically significant, transitional and perirenal which subsided spontaneously without intervention, 2 regressed back pain with analgesic therapy. No patient developed ablation related infection such as renal abscess, pyelonephritis or sepsis. Percutaneous cryoablation of small renal cell carcinoma was accomplished safely in all 32 cases. No episodes of urinary extravasation, significant gross hematuria or urinary obstruction occurred. No other major operative complications were encountered. Median hospital stay was 2 days (IQR: 13). Median follow-up after the procedure was 30 months (IQR: 8-59); In none case, incomplete treatment and local relapse or metastases at CT abdomen-pelvis with contrast medium at 3,6 and 12 months was detected. None of the 32 patients had clinical evidence of recurrent disease (confirmed with no enhancement within resolved tumor masses) at last follow-up. Median tumor size before treatment was $3.16 \mathrm{~cm}$ (IQR: 1.6 to 5.5 ) (Figure 1).

After treatment, cryo-lesions size continued to decline over time. The relative reduction of the lesion size compared to preoperative values was $21.77 \%$ at 3-month follow-up (median size: $2.47 \mathrm{~cm}$; IQR: 0.9 to 4.8 ), 31.93\% at six-month follow-up (median size: $2.2 \mathrm{~cm}$; IQR: 0.6 to $4.5 \mathrm{~cm}$ ) and $45.91 \%$ at one-year follow-up (median size: $1.71 \mathrm{~cm}$; IQR: 0.6 to $3.5 \mathrm{~cm}$ ).

Figures 2 and 3 show the gradual decrease
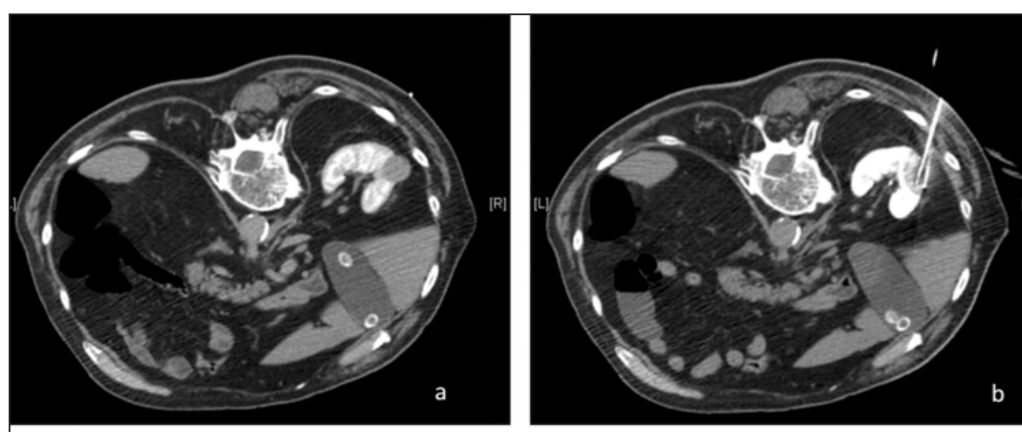

in the larger diameter of the lesions and the corresponding decrease in terms of percentage compared to the initial dimensions.

The temporary increase in diameter of the cryolesions immediately following cryoablation is most likely due to edema in the tissue surrounding the tumor. The levels of postoperative creatinine were identical with preoperative levels.
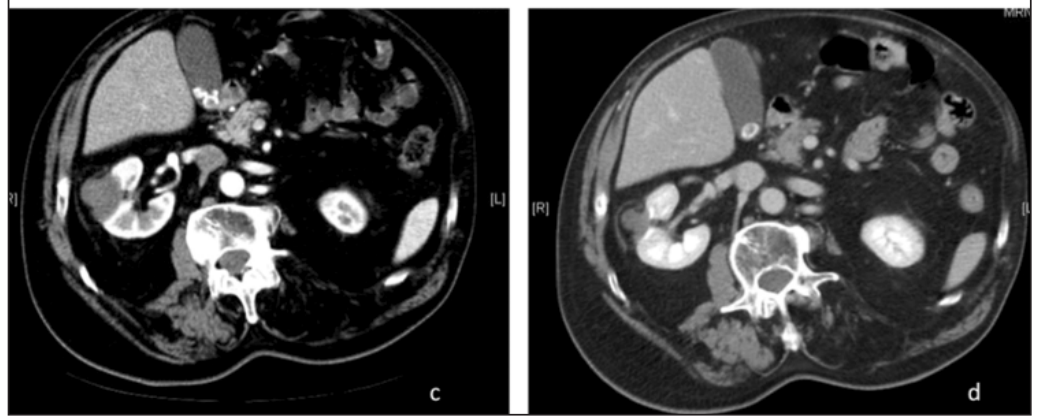

\section{Figure 1.}

Tumor in the middle right renal before cryoablation a); subsequent treatment b); lesion after 6 months c); lesion after 1-year d). 


\section{Figure 2.}

Tumor size variation evaluated using CT scans at 3, 6, 12 months of follow-up. The figure represents tumor size of 24 patients with a follow-up of at least 12 months. Each group of three columns represents a patients and age is specified below.

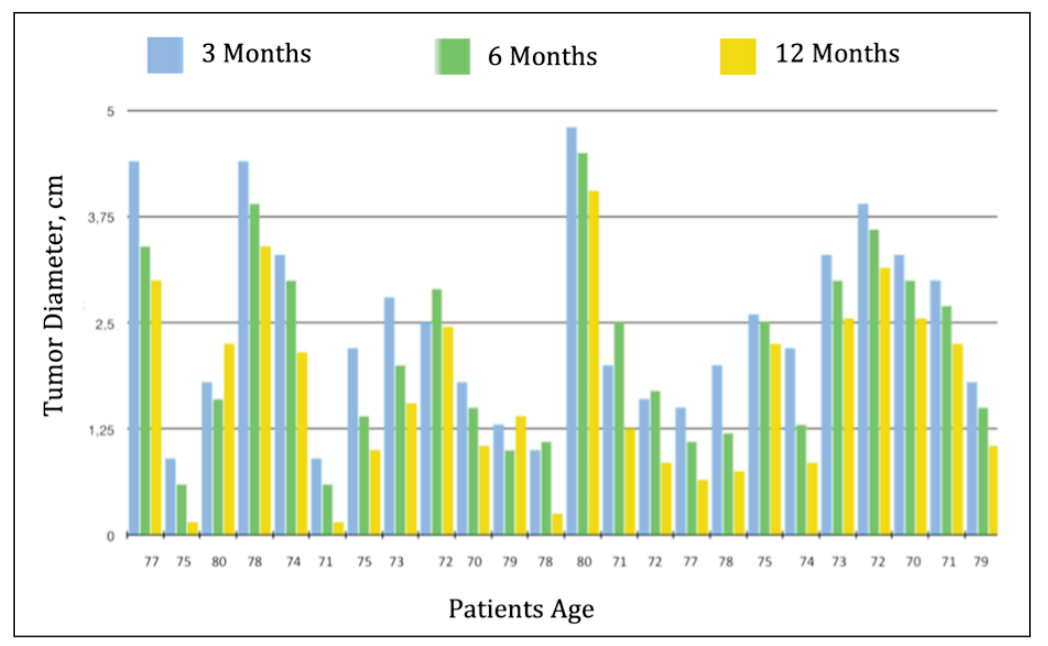

\section{Figure 3.}

Percentage variations in the major diameter of injuries.

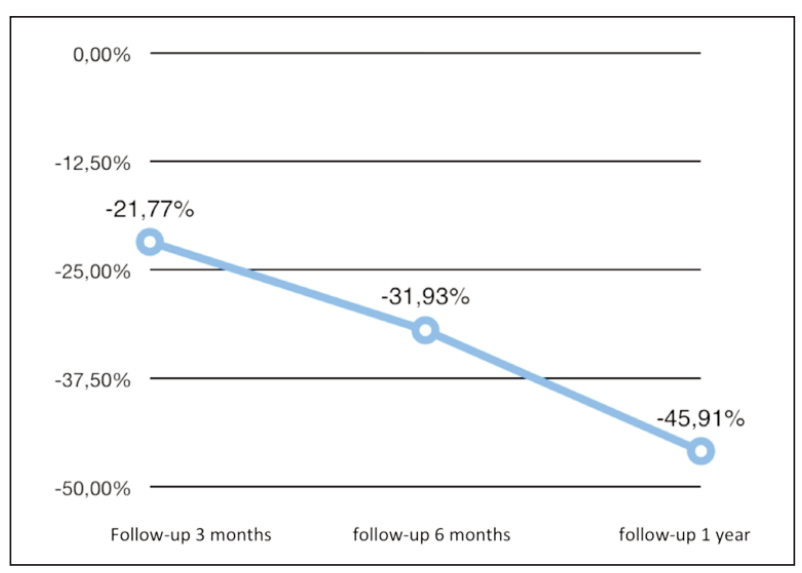

\section{Discussion}

The choice between conservative or radical treatment for organ confined RCC in men over 70 years is a debated topic. In the last decades, the standard of care has slowly been expanding from radical nephrectomy to include robotic partial nephrectomy, laparoscopic nephrectomy, laparoscopic ablation and finally percutaneous ablation $(14,15)$. Several questions such as efficacy and safety treatment, life expectancy, and cancer-specific survival advantage need to be taken into account. Image guided percutaneous ablation is especially attractive for patients with small renal masses and a solitary kidney or multiple tumors, non-surgical candidates, pre dialysis patients and patients with diseases that may cause multiple RCC as von Hippel-Lindau disease or hereditary RCC. Limited data are currently available in literature for assessing the role of percutaneous renal cryoablation. Silverman et al. described a large number of percutaneous renal cryoablations under general anesthesia with MRI guidance (16). Image guidance for percutaneous renal cryoablation is alternated between MRI, CT, and ultrasound (US). In the case of MRI, the question is that system is expensive and limited in use but the ability of real time guidance and visualization of the iceball in multiple planes are the advantage, allowing the operator to achieve the safety margins of the cryolesion. Bassignari et al. reported the practicability of US-guided percutaneous renal cryoablation to aim for future performance of this treatment by a urologist alone without the interventional radiologist because urologists have ultrasound experience (17). Results of this study from our cohort of patients showed that CT-guided percutaneous cryoablation resulted in complete response for lesions as large as $4 \mathrm{~cm}$. In all cases, it has a high efficacy rate, and its few complications are not only similar to those of other treatment modalities, but also appear to be reversible. Percutaneous cryoablation with use of CT has the benefit of delaying/avoiding the end-stage renal disease by causing less renal injury. Another advantage is to perform percutaneous cryoablation under spinal anesthesia avoiding the major complications or side effects that could derive from a general anesthesia considering that many patients have plurimorbidity as well as advanced age. Other advantages of percutaneous cryoablation are faster recovery, repeatable, and lower cost. Among all percutaneous ablative techniques, cryoablation has gained particular interest for small kidney cancer for painless during the treatment and it provides real-time image feedback of the ablation zone, especially when performed with CT guidance.

Furthermore, cryoablation is less likely to result in significant treatment-related injury because the extracellular matrix is less permanently destroyed by freezing, thereby allowing for epithelial regrowth and repair. The study pointed out that percutaneous cryotherapy provides, in elderly patients with small kidney cancer, an acceptable efficacy and safety in the absence of recurrence of disease and risk of metastasis in the short to medium follow-up (median follow-up: 30 months). This result is particularly encouraging considering that Andrews et al. (18) highlights that there are no statistically significant differences in terms of local recurrence, metastases and risk of death from RCC among partial nephrectomy, radiofrequency ablation and cryoablation for cTla patients. Pierorazio et al. (8) shows similar cancer specific survival among partial nephrectomy and thermal ablations (radiofrequency and cryoablation), with some differences in renal functional and peri/postoperative outcomes that should be considered when choosing a management strategy, particularly in elderly patient. Kitley et al. demonstrated in cTla that cryotherapy had a lower overall survival than partial nephrectomy however at multivariable analisys adjusting for age and Charlson Comorbidity Index, the rates of survival for the two treatments were similar (19). Additionally, overall survival of patients based on tumor size prove similar rates of survival for tumors smaller than $2 \mathrm{~cm}$. To our knowledge, these data suggest that elderly men with small renal cancer can take advantage from this local treatment. This is of clinical relevance in view of the 
fact that, as mentioned above, partial and ever more radical nephrectomy is usually not offered to elderly patients due to the risk of treatment-related complications, though their occurrence, like for most surgical procedures, is much linked to case volume (20). At the end, our data is similar to those published by Atwell et al., who reported a technical success and efficacy rate of $95 \%$ with a median follow-up of 8 months (21). Several guidelines and commentaries have highlighted the need for longer follow-up of ablation patients. Our study has limitations: all patients were referred from a urology department so may have introduced a selection bias. Additionally, even though the behavior of RCC is unpredictable, it is generally as low-growing cancer, and longer follow-up would be required to confirm the high degree of efficacy we documented in the present study.

\section{Conclusions}

Published preliminary results have shown that imageguided percutaneous cryoablation for small renal cancer is probably as safe and effective as laparoscopic cryoablation. Results of this study confirm the very high efficacy rate and acceptable morbidity associated with this CT-guided percutaneous cryoablation. Nonetheless, CT-guided percutaneous cryoablation offers compelling advantages compared with other treatment modalities, particularly in elderly patients, and has shown high efficacy rates and low risk for minor/major complications.

\section{REFERENCES}

1. Jayson M, Sanders H. Increased incidence of serendipitously discovered renal cell carcinoma. Urology. 1998; 51:203-5.

2. Homma Y, Kawabe K, Kitamura T, et al. Increased incidental detection and reduced mortality in renal cancer--recent retrospective analysis at eight institutions. Int J Urol. 1995; 2:77-80.

3. Decastro GJ, McKiernan JM. Epidemiology, clinical staging, and presentation of renal cell carcinoma. Urol Clin North Am. 2008; 35:581-92.

4. Saad AM, Gad MM, Al-Husseini MJ, et al. Trends in renal-cell carcinoma incidence and mortality in the United States in the last 2 decades: A SEER-based study. Clin Genitourin Cancer. 2019; 17:46$57 \mathrm{e} 5$.

5. Bray F, Ferlay J, Soerjomataram I, et al. Global cancer statistics 2018: GLOBOCAN estimates of incidence and mortality worldwide for 36 cancers in 185 countries. CA Cancer J Clin. 2018; 68:394-424.

6. Falagario UG, Martini A, Pfail J, et al. Does race impact functional outcomes in patients undergoing robotic partial nephrectomy? Transl Androl Urol. 2020; 9:863-869

7. Martini A, Falagario UG, Cumarasamy S, et al. Defining risk categories for a significant decline in estimated glomerular filtration rate after robotic partial nephrectomy: implications for patient follow-up. Eur Urol Oncol. 2019:S2588-9311(19)30104-X

8. Pierorazio PM, Johnson MH, Patel HD, et al. Management of renal masses and localized renal cancer: systematic review and meta-analysis. J Urol. 2016; 196:989-99.

9. Ljungberg B, Albiges L, Bensalah K, et al. EAU Guidelines on Renal Cell Carcinoma 2018. European Association of Urology Guidelines 2018 Edition, Vol. presented at the EAU Annual
Congress Copenhagen 2018. Arnhem, The Netherlands European Association of Urology Guidelines Office, 2018.

10. Gill IS, Novick AC, Soble JJ, et al. Laparoscopic renal cryoablation: initial clinical series. Urology. 1998; 52:543-51.

11. Gill IS, Desai MM, Kaouk JH, et al. Laparoscopic partial nephrectomy for renal tumor: duplicating open surgical techniques. J Urol. 2002; 167:469-7.

12. Ukimura O, Kawauchi A, Fujito A, et al. Radio-frequency ablation of renal cell carcinoma in patients who were at significant risk. Int J Urol. 2004; 11:1051-7.

13. de la Rosette JJ, Opondo D, Daels FP, et al. Categorisation of complications and validation of the Clavien score for percutaneous nephrolithotomy. Eur Urol. 2012; 62:246-55.

14. Corongiu E, Grande P, Di Santo A, et al. Safety and efficacy of retroperitoneal sutureless zero ischemia laparoscopic partial nephrectomy for low nephrometry score masses. Arch Ital Urol Androl. 2019; 91:157-162.

15. Tiscione D, Cai T, Luciani LG, et al. Sutureless laparoscopic partial nephrectomy using fibrin gel reduces ischemia time while preserving renal function. Arch Ital Urol Androl. 2019; 91:30-34.

16. Silverman SG, Tuncali K, vanSonnenberg E, et al. Renal tumors: MR imaging-guided percutaneous cryotherapy--initial experience in 23 patients. Radiology. 2005; 236:716-24.

17. Bassignani M, Moore Y, Watson L, Theodorescu D. Pilot experience with real-time ultrasound guided percutaneous renal mass cryoablation. J Urol. 2004; 171:1620-3.

18. Andrews JR, Atwell T, Schmit G, et al. Oncologic Outcomes Following Partial Nephrectomy and Percutaneous Ablation for cT1 Renal Masses. Eur Urol. 2019; 76:244-51.

19. Kitley W, Sulek J, Sundaram C, Bahler CD. Treatment trends and long-term survival associated with cryotherapy and partial nephrectomy for small renal masses in the National Cancer Database using propensity score matching. J Endourol. 2019; 33:408-14.

20. Kandasami SV, Mamoulakis C, El-Nahas AR, et al. Impact of case volume on outcomes of ureteroscopy for ureteral stones: the clinical research office of the endourological society ureteroscopy global study. Eur Urol. 2014; 66:1046-51

21. Atwell TD, Farrell MA, Callstrom MR, et al. Percutaneous cryoablation of 40 solid renal tumors with US guidance and CT monitoring: initial experience. Radiology. 2007; 243:276-83.

\section{Correspondence}

Oscar Selvaggio, MD oscarsel@libero.it

Giovanni Silecchia, MD

Ugo Giovanni Falagario, MD

Giuseppe Carrieri, MD

Luigi Cormio, MD

University of Foggia, Department of Urology and Organ Transplantation Viale Pinto 1, 71100 Foggia (Italy)

Matteo Gravina, MD

Luca Macarini, MD

University of Foggia, Department of Radiology, Foggia, Italy

Giovanni Stallone, MD

University of Foggia, Department of Nephrology, Foggia, Italy 